PROCEEDINGS OF THE

AMERICAN MATHEMATICAL SOCIETY

Volume 124, Number 12, December 1996, Pages 3641-3646

S 0002-9939(96)03865-8

\title{
ON THE PLURICANONICAL MAP OF THREEFOLDS OF GENERAL TYPE
}

\author{
DONG-KWAN SHIN
}

(Communicated by Eric M. Friedlander)

\begin{abstract}
Let $X$ be a smooth minimal threefold of general type and let $n$ be an integer $>1$. Assume that the image of the pluricanonical map $\Phi_{n}$ of $X$ is a curve. Then a simple computation shows that $n$ is necessarily 2 or 3 . When $n=2$ with a numerical condition or when $n=3$, we obtain two inequalities $\chi\left(\mathcal{O}_{X}\right) \leq \min \left\{-1,2-2 q_{1}\right\}$ and $q_{1} \leq \frac{3}{14} K_{X}{ }^{3}+1$, where $q_{1}$ is the irregularity of $X$ and $\chi\left(\mathcal{O}_{X}\right)$ is the Euler characteristic of $X$.
\end{abstract}

Throughout this paper, we are working over the complex number field $\mathbf{C}$.

In this paper, we have studied the case that the image $C$ of the pluricanonical map $\Phi_{n}$ is a curve for an integer $n>1$. In this case, a simple calculation shows $n=2$ or 3 . Resolve the base locus of $\Phi_{n}$. Then we have two terms ' $b$ ' and ' $c$ ' explained just below Proposition 1 . When $n=2$ with additional numerical conditions or when $n=3$, we may have some information about $\Phi_{n}$ from these two terms, which are explained in Corollary of Proposition 2 and Proposition 3. Using this information, we obtain two inequalities $\chi\left(\mathcal{O}_{X}\right) \leq \min \left\{-1,2-2 q_{1}\right\}$ and $q_{1} \leq \frac{3}{14} K_{X}{ }^{3}+1$, which are given in Theorems 2 and 3 .

Now, let's set up our notations. Let $X$ be a smooth projective variety and let $D$ be a divisor on $X$. Denote by $K_{X}$ the canonical divisor of $X$. Denote by $\Phi_{n}$ the rational map associated to the complete linear system $\left|n K_{X}\right|$. Denote by $h^{i}\left(X, \mathcal{O}_{X}(D)\right)$ the dimension of $H^{i}\left(X, \mathcal{O}_{X}(D)\right)$. Let $\mathrm{Bs}|D|$ mean the base locus of $|D|$. Let's denote the genus of $X$ by $p_{g}(X)$ and $h^{i}\left(X, \mathcal{O}_{X}\right)$ by $q_{i}(X)$. (or simply $p_{g}$ and $q_{i}$ unless there is any confusion.) Denote by $\sim$ the linear equivalence and by $\equiv$ the numerical equivalence. For a real number $r,[r]$ means the greatest among the integers less than or equal to $r$.

Theorem 1 (Kawamata-Viehweg vanishing theorem). Let $X$ be a nonsingular projective variety. If $D$ is a nef and big divisor on $X$, then $H^{i}\left(X, \mathcal{O}_{X}\left(K_{X}+D\right)\right)=0$ for all $i>0$.

For a reference, see KMM [5].

Lemma. Let $X$ be a smooth projective threefold, and let $D$ be a divisor on $X$. Then we have the following:

Received by the editors June 12, 1995.

1991 Mathematics Subject Classification. Primary 14E05, 14J30.

Key words and phrases. Threefold of general type, pluricanonical map, fiber space, Euler characteristic.

This paper is supported by KOSEF and Dae-Yang Foundation. 
(a) $\chi\left(\mathcal{O}_{X}(D)\right)=D^{3} / 6-K_{X} \cdot D^{2} / 4+D \cdot\left(K_{X}^{2}+c_{2}\right) / 12+\chi\left(\mathcal{O}_{X}\right)$, where $c_{2}$ is the second Chern class of $X$. Moreover $\chi\left(\mathcal{O}_{X}\right)=-c_{2} \cdot K_{X} / 24$.

(b) $K_{X} \cdot D^{2}$ is even.

(c) $p_{n}: \stackrel{\text { def }}{=} h^{0}\left(X, \mathcal{O}_{X}\left(n K_{X}\right)\right)=\frac{n(n-1)(2 n-1)}{12} K_{X}{ }^{3}+(1-2 n) \chi\left(\mathcal{O}_{X}\right)$ for $n \geq 2$ and $\chi\left(\mathcal{O}_{X}\right)<0$ when $K_{X}$ is nef and big.

Proof. (a) comes from the Riemann-Roch theorem.

(b) comes from the following:

$$
\chi\left(\mathcal{O}_{X}(D)\right)+\chi\left(\mathcal{O}_{X}(-D)\right)=-K_{X} \cdot D^{2} / 2+2 \chi\left(\mathcal{O}_{X}\right) \in \mathbf{Z} .
$$

(c) comes from (a) and the Kawamata-Viehweg vanishing theorem. We have $\chi\left(\mathcal{O}_{X}\right)<0$ since $-c_{2} \cdot K_{X} \leq-K_{X}^{3} / 3$. (See Miyaoka [8].)

Proposition 1. Let $C$ be a nondegenerate curve of degree a in $\mathbf{P}^{n}$. Then we have

(a) If $n \leq a<2 n$, then $p_{g}(C) \leq a-n$.

(b) If $2 n \leq a$, then $p_{g}(C) \leq \frac{m(m-1)}{2}(n-1)+m r$, where $m=\left[\frac{a-1}{n-1}\right]$ and $a-1=m(n-1)+r$.

Proof. See Griffiths \& Harris [2], p. 253.

We are going to use the following notations in the rest of this paper.

Let $X$ be a smooth projective threefold of general type with $K_{X}$ nef. Let $n$ be a positive integer $\geq 2$.

Suppose that the dimension of the image $C$ of $\Phi_{n}$ is 1 .

Let $\left|n K_{X}\right|=|M|+Z$, where $|M|$ and $Z$ are the moving part and fixed part of $\left|n K_{X}\right|$ respectively. Let $f: X^{\prime} \rightarrow X$ be the resolution of the base locus of $\Phi_{n}$ if $\mathrm{Bs}\left|n K_{X}\right| \neq \emptyset$, i.e., $f$ is a succession of blow-ups along nonsingular centers of codim $\geq 2$ in the base locus such that $g=\Phi_{n} \circ f$ is a morphism. Let $g=k \circ h$ be the Stein factorization. Observe that $C^{\prime}$ is normal and hence smooth.

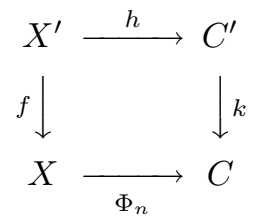

Let $a$ be $\operatorname{deg} C$ in $\mathbf{P}^{p_{n}-1}$, and let $b=\operatorname{deg} k$. Recall that $C$ is a nondegenerate curve in $\mathbf{P}^{p_{n}-1}$. Let $S$ be the general fiber of $h$.

We have $K_{X^{\prime}}=f^{*}\left(K_{X}\right)+E^{\prime}$ and $\left|f^{*} n K_{X}\right|=\left|M^{\prime}\right|+Z^{\prime}$, where $E^{\prime}$ is the ramification divisor of $f$ supported on the exceptional locus of $f$ and $Z^{\prime}$ is the fixed part of $\left|f^{*} n K_{X}\right|$. We have $M^{\prime} \equiv a b S$. Since $f^{*} K_{X}$ is nef, $f^{*} K_{X}{ }^{2} \cdot Z^{\prime} \geq 0$. Hence

$$
n K_{X}{ }^{3}=n f^{*} K_{X}{ }^{3}=\left(a b S+Z^{\prime}\right) f^{*} K_{X}{ }^{2} \geq a b f^{*} K_{X}{ }^{2} \cdot S .
$$

Let $c=f^{*} K_{X}{ }^{2} \cdot S$. Since $f^{*} K_{X}$ is nef and big, and $S$ is nef and not numerically equivalent to 0 , we have $f^{*} K_{X}{ }^{2} \cdot S \geq 1$. Hence

$$
\frac{n K_{X}^{3}}{b c} \geq a \geq p_{n}-1
$$

From the inequality (1) and Lemma, the image of $\Phi_{n}$ can be a curve only when $n$ is 2 or 3 . Moreover, we have $1 \leq b c \leq 3$ and in particular, $b c=1$ when $n=3$. 
Proposition 2 (cf. Matsuki [7]). If $\operatorname{dim} \operatorname{Im} \Phi_{n}=1$, then $S$ is a surface of general type and $S^{\prime}$ has $K_{S^{\prime}}{ }^{2}=f^{*} K_{X}{ }^{2} \cdot S$, where $\pi: S \rightarrow S^{\prime}$ is a morphism of $S$ to its minimal surface $S^{\prime}$.

Proof. The easy addition formula ' $\kappa(X) \leq \kappa(S)+\operatorname{dim} C$ ' implies that $S$ is the surface of general type, where $\kappa(X)$ means the Kodaira dimension of $X$. (For a reference about easy addition formula, see Ueno [9].) We have that $n K_{X} \equiv a b D+Z$ and $f^{*} a b D \equiv a b S+E$, where $D=f_{*} S$ and $E$ is an effective divisor supported on the exceptional locus of $f$. So,

$$
n f^{*} K_{X}^{2} \cdot S=n K_{X}^{2} \cdot D=(a b D+Z) \cdot K_{X} \cdot D \geq a b K_{X} \cdot D^{2} .
$$

Since $D^{2}$ is an effective 1 -cycle and $K_{X}$ is nef, $K_{X} \cdot D^{2} \geq 0$. If $K_{X} \cdot D^{2} \neq 0$, then $n f^{*} K_{X}{ }^{2} \cdot S \geq 2 a \geq 2\left(p_{n}-1\right)$ since $K_{X} \cdot D^{2}$ is even. This inequality holds true only when $n=2, K_{X}{ }^{3}=2, \chi\left(\mathcal{O}_{X}\right)=-1$ and $f^{*} K_{X}{ }^{2} \cdot S=3$. (Recall that $2 \leq n \leq 3$ and $1 \leq c \leq 3$.) But it does not satisfy the inequality (1). Hence $K_{X} \cdot D^{2}=0$. So

$$
\begin{aligned}
0=(a b D)^{2} \cdot K_{X} & =f^{*} a b D \cdot f^{*} a b D \cdot f^{*} K_{X} \\
& =(a b S+E) \cdot f^{*} a b D \cdot f^{*} K_{X} \\
& =a b S \cdot f^{*} a b D \cdot f^{*} K_{X} \\
& =a b S \cdot(a b S+E) \cdot f^{*} K_{X} \\
& =a b S \cdot E \cdot f^{*} K_{X} .
\end{aligned}
$$

Hence we have $S \cdot E \cdot f^{*} K_{X}=0$. Let $\left\{E_{i}\right\}$ be the irreducible components of $E$. Clearly $S \cdot E_{i} \cdot f^{*} K_{X}=0$ for each $i$. By the way of taking $f, \operatorname{supp}(E)=\operatorname{supp}\left(E^{\prime}\right)$. Hence we have $S \cdot E^{\prime} \cdot f^{*} K_{X}=0$, since $S \cdot E_{i} \cdot f^{*} K_{X}=0$ for each $i$.

Applying the Hodge index theorem to $S$, we have that $\left(\left.E^{\prime}\right|_{S}\right)^{2}=S \cdot E^{\prime 2} \leq 0$ since $\left(\left.f^{*} K_{X}\right|_{S}\right)^{2}=f^{*} K_{X}{ }^{2} \cdot S \geq 1$. We have $\left.f^{*} K_{X}\right|_{S}+\left.E^{\prime}\right|_{S} \sim K_{S} \sim \pi^{*} K_{S^{\prime}}+L$, where $L$ is an effective divisor supported on the exceptional locus of $\pi$. Hence the uniqueness of the Zariski decomposition implies that $\left.f^{*} K_{X}\right|_{S} \sim \pi^{*} K_{S^{\prime}}$.

Corollary. If $f^{*} K_{X}{ }^{2} \cdot S=1$, then the minimal surface $S^{\prime}$ of $S$ has $K_{S^{\prime}}{ }^{2}=1$, $q(S)=0$ and $1 \leq p_{g}(S) \leq 2$.

Proof. $K_{S^{\prime}}{ }^{2}=\left.f^{*} K_{X}\right|_{S}{ }^{2}=1$ by Proposition 2. Since $K_{S^{\prime}}{ }^{2}=1$, we have that $q(S)=0$ and $p_{g}(S) \leq 2$. (For a reference, see Bombieri [1], p. 212.) Consider the exact sequence

$$
0 \rightarrow \mathcal{O}_{X^{\prime}}\left(K_{X^{\prime}}\right) \rightarrow \mathcal{O}_{X^{\prime}}\left(K_{X^{\prime}}+M^{\prime}\right) \rightarrow \stackrel{a b}{\oplus} \mathcal{O}_{S}\left(K_{S}\right) \rightarrow 0 .
$$

The above exact sequence shows that $p_{g}(S) \neq 0$ since $M^{\prime}$ is not fixed.

We have two facts from the condition $b c=1$. The first one is that $C^{\prime}$ is birational to the image $C$ of the pluricanonical map $\Phi_{n}$. So we may assume that $C$ is smooth since the terms we are interested in are birational invariants. The second one is that a general fiber of $g$ is a surface of general type with its irregularity 0 from Corollary of Proposition 2.

Proposition 3. Suppose that $b c=1$. Then we have that $1 \leq p_{g}(S) \leq 2$ and $\chi\left(\mathcal{O}_{X}\right) \leq\left(p_{g}(S)+1\right)\left(1-q_{1}\right)$. 
Proof. We have the fiber space $g: X^{\prime} \rightarrow C$ with connected fiber since $b c=1$. By Corollary of Proposition $2, q(S)=0$ and $1 \leq p_{g}(S) \leq 2$. We have $R^{1} g_{*} K_{X^{\prime}}=0$ since $q(S)=h^{1}\left(S, \mathcal{O}_{S}\left(K_{S}\right)\right)=0$. By spectral sequence, we have that

$$
\begin{aligned}
& p_{g}=h^{0}\left(X, \mathcal{O}_{X}\left(K_{X}\right)\right)=h^{0}\left(C, g_{*} K_{X^{\prime}}\right), \\
& q_{2}=h^{1}\left(X, \mathcal{O}_{X}\left(K_{X}\right)\right)=h^{1}\left(C, g_{*} K_{X^{\prime}}\right), \\
& q_{1}=h^{2}\left(X, \mathcal{O}_{X}\left(K_{X}\right)\right)=h^{0}\left(C, R^{2} g_{*} K_{X^{\prime}}\right) .
\end{aligned}
$$

Since $R^{2} g_{*} K_{X^{\prime}}=K_{C}, q_{1}=p_{g}(C)$. (For a reference, see Kollár [6].) It is known that $g_{*} K_{X^{\prime} / C} \stackrel{\text { def }}{=} g_{*}\left(K_{X^{\prime}} \otimes g^{*} K_{C}{ }^{-1}\right)$ is semipositive and locally free of rank $p_{g}(S)$. So $\operatorname{deg} g_{*} K_{X^{\prime} / C} \geq 0$. (See Kawamata [4].)

$$
\begin{aligned}
h^{0}\left(C, g_{*} K_{X^{\prime}}\right)-h^{1}\left(C, g_{*} K_{X^{\prime}}\right) & =\operatorname{deg} g_{*} K_{X^{\prime}}+p_{g}(S)\left(1-p_{g}(C)\right) \\
& =\operatorname{deg} g_{*} K_{X^{\prime} / C}+p_{g}(S)\left(p_{g}(C)-1\right) \\
& \geq p_{g}(S)\left(p_{g}(C)-1\right) .
\end{aligned}
$$

So $p_{g}-q_{2} \geq p_{g}(S)\left(q_{1}-1\right)$. Hence $-\chi\left(\mathcal{O}_{X}\right)=p_{g}-q_{2}+q_{1}-1 \geq\left(p_{g}(S)+1\right)\left(q_{1}-1\right)$. Now we have $\chi\left(\mathcal{O}_{X}\right) \leq\left(p_{g}(S)+1\right)\left(1-q_{1}\right)$.

Theorem 2. If $\operatorname{dim} \operatorname{Im} \Phi_{3}=1$, then the following hold:

(a) $-\frac{1}{10} K_{X}{ }^{3}-\frac{1}{5} \leq \chi\left(\mathcal{O}_{X}\right) \leq \min \left\{-1,2-2 q_{1}\right\}$. Moreover, $K_{X}{ }^{3} \geq 8$.

(b) $q_{1} \leq \frac{1}{22} K_{X}{ }^{3}+1$.

Proof. The fact $b c=1$ is given just above Proposition 2. If $a \geq 2\left(p_{3}-1\right)$, then the inequality (1) implies $3 K_{X}^{3} / 2 \geq p_{3}-1$. But it is impossible since $p_{3}=$ $\frac{5}{2} K_{X}{ }^{3}-5 \chi\left(\mathcal{O}_{X}\right)$. So $b c=1$ and $a<2\left(p_{3}-1\right)$. Hence Lemma and Proposition 3 imply $\chi\left(\mathcal{O}_{X}\right) \leq \min \left\{-1,2-2 q_{1}\right\}$. From the inequality (1), we have $-\frac{1}{10} K_{X}{ }^{3}-\frac{1}{5} \leq$ $\chi\left(\mathcal{O}_{X}\right)$. Moreover, since $\chi\left(\mathcal{O}_{X}\right) \leq-1, K_{X}{ }^{3} \geq 8$.

For (b), by Proposition $1, p_{g}(C) \leq a-\left(p_{3}-1\right)$ since the degree $a$ of $C$ is less than $2\left(p_{3}-1\right)$. Recall that we have $q_{1}=p_{g}(C)$ in the proof of Proposition 3 . We already know $a \leq 3 K_{X}{ }^{3}$ from (1). Hence

$$
q_{1}=p_{g}(C) \leq a-\left(p_{3}-1\right) \leq 3 K_{X}{ }^{3}-p_{3}+1 \leq 1 / 2 K_{X}^{3}+5 \chi\left(\mathcal{O}_{X}\right)+1 .
$$

From (a), $q_{1} \leq 1 / 2 K_{X}{ }^{3}+5\left(2-2 q_{1}\right)+1$. Hence we have $q_{1} \leq \frac{1}{22} K_{X}{ }^{3}+1$.

Theorem 3. Suppose that $\operatorname{dim} \operatorname{Im} \Phi_{2}=1$. If $b c=1$, then we have the following:

(a) $\chi\left(\mathcal{O}_{X}\right) \leq \min \left\{-1,2-2 q_{1}\right\}$.

(b) $q_{1} \leq \frac{3}{14} K_{X}{ }^{3}+1$.

Proof. Lemma and Proposition 3 imply (a) clearly.

For (b), we already know $p_{2}-1 \leq a \leq 2 K_{X}{ }^{3}$ from (1). Hence it is enough to consider the following two cases:

Case 1. $p_{2}-1 \leq a<2\left(p_{2}-1\right)$. 
Since $p_{2}-1 \leq a<2\left(p_{2}-1\right)$, Proposition 1 implies that $p_{g}(C) \leq a-\left(p_{2}-1\right)$. Since $b c=1$, the proof of Proposition 3 implies that $q_{1}=p_{g}(C)$ and $\chi\left(\mathcal{O}_{X}\right) \leq 2-2 q_{1}$.

$$
\begin{aligned}
q_{1}=p_{g}(C) & \leq a-\left(p_{2}-1\right) \\
& \leq 2 K_{X}^{3}-p_{2}+1 \\
& =\frac{3}{2} K_{X}{ }^{3}+3 \chi\left(\mathcal{O}_{X}\right)+1 \\
& \leq \frac{3}{2} K_{X}{ }^{3}+3\left(2-2 q_{1}\right)+1 .
\end{aligned}
$$

Hence we have $q_{1} \leq \frac{3}{14} K_{X}{ }^{3}+1$.

Case 2. $2\left(p_{2}-1\right) \leq a \leq 2 K_{X}{ }^{3}$.

Since $2\left(p_{2}-1\right) \leq \bar{a} \leq 2 K_{X}{ }^{3}$, Proposition 1 implies that

$$
p_{g}(C) \leq \frac{m(m-1)}{2}\left(p_{2}-2\right)+m r
$$

where $m=\left[\frac{a-1}{p_{2}-2}\right]$ and $a-1=m\left(p_{2}-2\right)+r$.

So, let's compute $m$. Since $2\left(p_{2}-1\right) \leq a \leq 2 K_{X}{ }^{3}$, we have that

$$
\frac{2\left(p_{2}-1\right)-1}{p_{2}-2} \leq \frac{a-1}{p_{2}-2} \leq \frac{2 K_{X}{ }^{3}-1}{p_{2}-2} .
$$

If we modify the above inequalities, we have that

$$
2+\frac{1}{K_{X}{ }^{3} / 2-3 \chi\left(\mathcal{O}_{X}\right)-2} \leq \frac{a-1}{p_{2}-2} \leq 4+\frac{12 \chi\left(\mathcal{O}_{X}\right)+7}{K_{X}{ }^{3} / 2-3 \chi\left(\mathcal{O}_{X}\right)-2} .
$$

Since $\chi\left(\mathcal{O}_{X}\right)<0$, we have that

$$
\frac{1}{K_{X}{ }^{3} / 2-3 \chi\left(\mathcal{O}_{X}\right)-2}>0 \text { and } \frac{12 \chi\left(\mathcal{O}_{X}\right)+7}{K_{X}{ }^{3} / 2-3 \chi\left(\mathcal{O}_{X}\right)-2}<0 .
$$

Hence we have $m=\left[\frac{a-1}{p_{2}-2}\right]=2$ or 3 .

When $m=2$, we have that

$$
\begin{aligned}
q_{1}=p_{g}(C) & \leq \frac{2 \cdot 1}{2}\left(p_{2}-2\right)+2 r \\
& \leq\left(p_{2}-2\right)+2\left(a-1-2\left(p_{2}-2\right)\right) \\
& \leq 2 a-3 p_{2}+4 \\
& \leq 4 K_{X}{ }^{3}-3\left(\frac{K_{X}{ }^{3}}{2}-3 \chi\left(\mathcal{O}_{X}\right)\right)+4 \\
& \leq \frac{5}{2} K_{X}{ }^{3}+9 \chi\left(\mathcal{O}_{X}\right)+4 \\
& \leq \frac{5}{2} K_{X}{ }^{3}+9\left(2-2 q_{1}\right)+4 .
\end{aligned}
$$

Hence $q_{1} \leq \frac{5}{38} K_{X}{ }^{3}+\frac{22}{19}$.

When $m=3$, similarly, we have that $q_{1} \leq \frac{3}{37} K_{X}{ }^{3}+\frac{45}{37}$.

Therefore, combining all inequalities about $q_{1}$, we have $q_{1} \leq \frac{3}{14} K_{X}{ }^{3}+1$. 
Remark. When $\operatorname{dim} \operatorname{Im} \Phi_{2}=1$, we assume the condition $b c=1$. At this moment, we don't have a necessary and sufficient condition to guarantee $b c=1$. But we have some cases which show $b c=1$.

Proposition 4. Suppose that $\operatorname{dim} \operatorname{Im} \Phi_{2}=1$. If $K_{X}{ }^{3}<p_{2}-1$, then we have that

(a) $b c=1$,

(b) $-K_{X}{ }^{3} / 2-1 / 3 \leq \chi\left(\mathcal{O}_{X}\right)<-K_{X}{ }^{3} / 6-1 / 3$.

Proof. Since $K_{X}{ }^{3}<p_{2}-1 \leq a$, we have $K_{X}{ }^{3}<p_{2}-1 \leq a \leq \frac{2 K_{X}{ }^{3}}{b c}$ from (1). Hence $b c$ must be 1 .

From (1), we have $-K_{X}{ }^{3} / 2-1 / 3 \leq \chi\left(\mathcal{O}_{X}\right)$. Since $K_{X}{ }^{3}<p_{2}-1$, we have $\chi\left(\mathcal{O}_{X}\right)<-K_{X}{ }^{3} / 6-1 / 3$. Combining these two inequalities, we have

$$
-K_{X}{ }^{3} / 2-1 / 3 \leq \chi\left(\mathcal{O}_{X}\right)<-K_{X}{ }^{3} / 6-1 / 3 .
$$

Proposition 5. Suppose that $\operatorname{dim} \operatorname{Im} \Phi_{2}=1$. If $2\left(p_{2}-1\right) \leq a$, then we have

(a) $b c=1$,

(b) $-K_{X}{ }^{3} / 6-1 / 3 \leq \chi\left(\mathcal{O}_{X}\right) \leq-1$.

Proof. Since $2\left(p_{2}-1\right) \leq a$, we have $2\left(p_{2}-1\right) \leq a \leq 2 K_{X}{ }^{3} / b c$ from (1). If $b c \geq 2$, then we have $2\left(p_{2}-1\right) \leq K_{X}{ }^{3}$. But, since $2\left(p_{2}-1\right)=K_{X}{ }^{3}-6 \chi\left(\mathcal{O}_{X}\right)-2>K_{X}{ }^{3}$, it is impossible. Hence $b c=1$.

From $2\left(p_{2}-1\right) \leq a \leq 2 K_{X}{ }^{3}$, we have $-K_{X}{ }^{3} / 6-1 / 3 \leq \chi\left(\mathcal{O}_{X}\right)$.

Remark. When $\operatorname{dim} \operatorname{Im} \Phi_{2}=1$, from (1), we may have the following three cases: $a \leq 2 K_{X}{ }^{3}<2\left(p_{2}-1\right), a \leq 2\left(p_{2}-1\right) \leq 2 K_{X}{ }^{3}$, and $2\left(p_{2}-1\right) \leq a \leq 2 K_{X}{ }^{3}$. The case we didn't cover here is the second one $p_{2}-1 \leq a \leq 2\left(p_{2}-1\right) \leq 2 K_{X}{ }^{3}$.

\section{REFERENCES}

1. E. Bombieri, Canonical models of surfaces of general type, Publ. Math. IHES, 42, (1973), 447-495. MR 47:6710

2. P. Griffiths and J. Harris, Principles of Algebraic Geometry, Wiley, New-York, 1978. MR 80b:14001

3. R.Hartshorne, Algebraic Geometry, Springer-Verlag, New-York-Heidelberg, 1977. MR $\mathbf{5 7 : 3 1 1 6}$

4. Y. Kawamata, Hodge theory and Kodaira dimension, Algebraic varieties and analytic varieties (ed. S. Iitaka), Adv. Stud. in Pure Math., vol. 1 (1983), 317-327. MR 85j:14012

5. Y.Kawamata, K.Matsuda, and K.Matsuki, Introduction to the minimal model problem, Algebraic Geometry, Sendai, 1985 (ed. T. Oda), Adv. Stud. in Pure Math., vol. 10 (1987), 283-360. MR 89e: 14015

6. J. Kollár, Higher direct images of dualizing sheaves I, Ann. of Math., 123, (1986), 11-42. MR 87c: 14038

7. K.Matsuki, On pluricanonical maps for 3-folds of general type, J. Math. Soc. Japan, 38 (1986), no. 2, 339-359. MR 87g:140436

8. Y. Miyaoka, The Chern classes and Kodaira dimension of a minimal variety, Algebraic Geometry, Sendai, 1985 (ed. T. Oda), Adv. Stud. in Pure Math., vol. 10 (1987), 449-476. MR 89k:14022

9. K. Ueno, Classification of algebraic varieties and compact complex spaces, Lecture Notes in Math., Springer-Verlag, Berlin, 1975. MR 58:22062

Department of Mathematics, Konkuk University, Seoul, 143-701, Korea

E-mail address: shindk@cs.sejong.ac.kr 